\title{
Heartbeat: delayed and inadequate treatment of acute coronary syndromes during the COVID-19 pandemic
}

doi:10.1136/heartjnl-2022-320938

The early phase of the COVID-19 pandemic was associated with fewer patients receiving primary percutaneous coronary intervention (PPCI) and a higher hospital mortality for acute myocardial infarction in Europe. In this issue of Heart, De Luca and colleagues ${ }^{1}$ report the final results of the International Study on Acute Coronary Syndromes-ST Elevation Myocardial Infarction (ISACS-STEMI) Registry which included over 16 thousand patients presenting March to June 2020 at 109 high-volume PPCI centres in Europe, Latin America, South-East Asia and North Africa. Overall, there was a lower rate of PPCI in 2020 compared with 2019 with marked geographical heterogeneity; but no association between PPCI volumes and peak COVID-19 case or mortality rates (figure 1). The reduction in PPCI was greatest in older adults (age $>75$ years). Compared to 2019, the COVID-19 pandemic was associated with longer door-to-balloon times, total ischaemia time and higher in-hospital $(6.5 \%$ vs $5.3 \%, \mathrm{p}<0.001)$ and 30 -day $(8 \%$ vs $6.5 \%, \mathrm{p}=0.001)$ mortality.

In the accompanying editorial, Cammann and Templin ${ }^{2}$ discuss the potential reasons for fewer patients presenting with an acute coronary syndrome during the pandemic, pointing out that this represents a failure to diagnosis and treat patients appropriately, not a reduction in disease prevalence. Delayed treatment or suboptimal intervention for acute coronary syndromes is likely to result in long-term detrimental consequences (figure 2). For those of us who trained in the early 1980s, the prevalence of postmyocardial infarction complications such as ventricular septal or papillary muscle rupture, ventricular thrombus, ischaemic cardiomyopathy, and arrhythmias is starting to look like the 'not-so-good old days'.

Division of Cardiology, University of Washington, Seattle, Washington, USA

Correspondence to Professor Catherine M Otto, Division of Cardiology, University of Washington, Seattle, WA 98195, USA; cmotto@uw.edu
Catherine M Otto

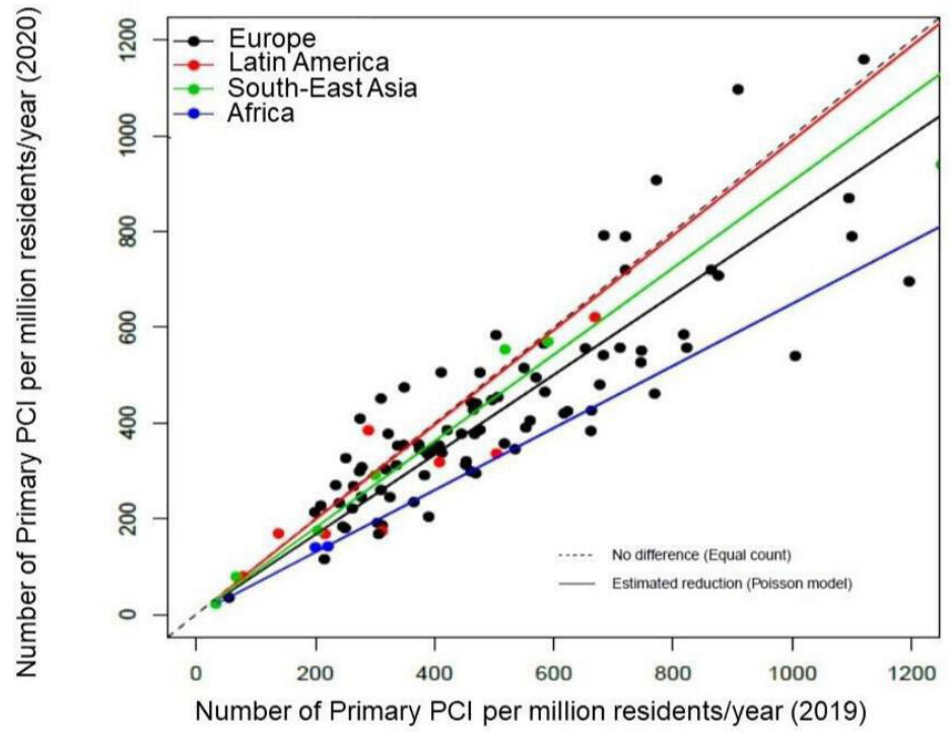

Figure 1 Results of Poisson regression analysis on the relationship between the number of primary PCI per million of residents per year in 2020 versus the number in 2019, according to continent. $\mathrm{PCl}$, percutaneous coronary intervention.
In patients with cardiac sarcoidosis (CS), improved prediction of the risk of heart failure and sudden death would allow individualised therapy. In a consecutive series of 61 patients with CS and abnormal ${ }^{8}$ F-fluorodeoxyglucose $\left({ }^{18} \mathrm{~F}-\mathrm{FDG}\right)$ accumulation in the heart, followed for a median of 46 months, independent predictors or sustained ventricular tachycardia (N-12) or

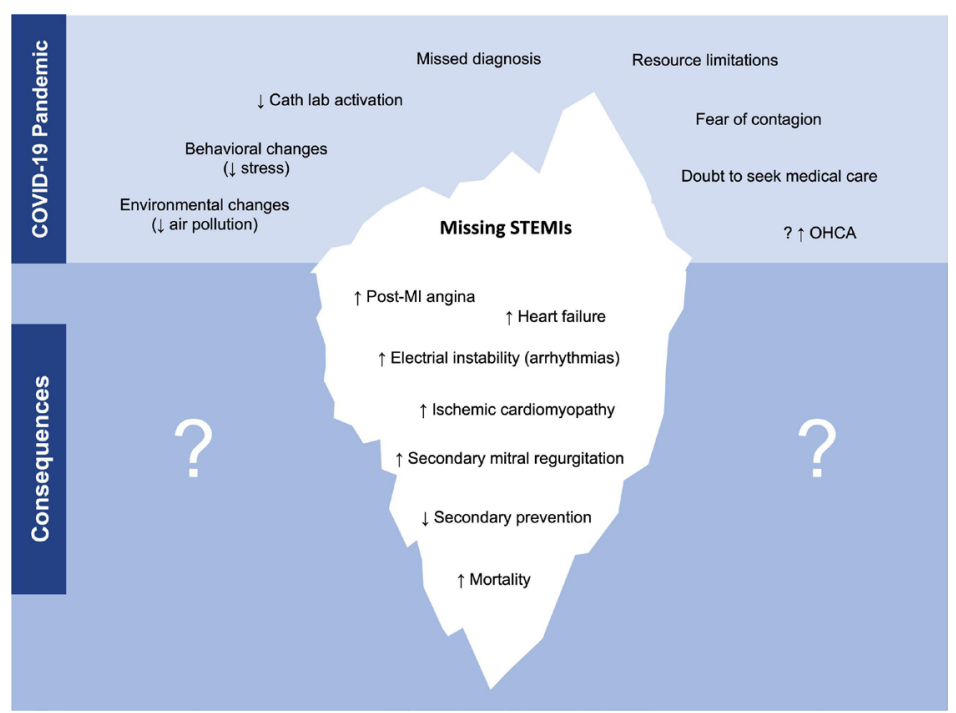

Figure 2 Potential causes and consequences of the missing STEMls during the COVID-19 pandemic. Summary of proposed hypothesis which may have contributed to the drop in STEMI cases during the COVID-19 pandemic (top) and potential consequences arising from it (bottom). $\mathrm{MI}$, myocardial infarction; OHCA, out-of-hospital cardiac arrest; STEMI, ST-segment elevation myocardial infarction. 
A. SVT/SCD (including patients with VT history)

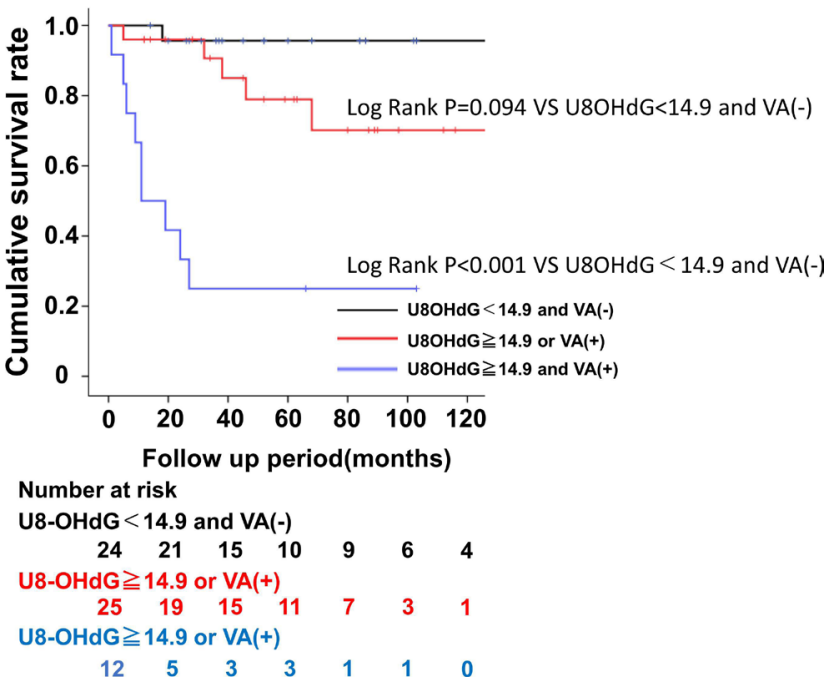

Figure 3 Risk stratification based on U-8-OHdG and ventricular aneurysm (VA). Patients showing $\mathrm{U}-8-\mathrm{OHdG}$ concentration $\geq 14.9 \mathrm{ng} / \mathrm{mg} \cdot \mathrm{Cr}$ and presence of VA had a significantly highest risk of sVT/SCD (log-rank, $\mathrm{p}<0.001$ vs patients with neither, events per 100 patient-year, 31.0 ). SCD, sudden cardiac death; sVT, sustained ventricular tachycardia; U-8-OHdG, urinary 8-hydroxy-2'deoxyguanosine; VA, ventricular aneurysm; VT, ventricular tachycardia.

\section{General considerations regarding sTR therapy strategies}

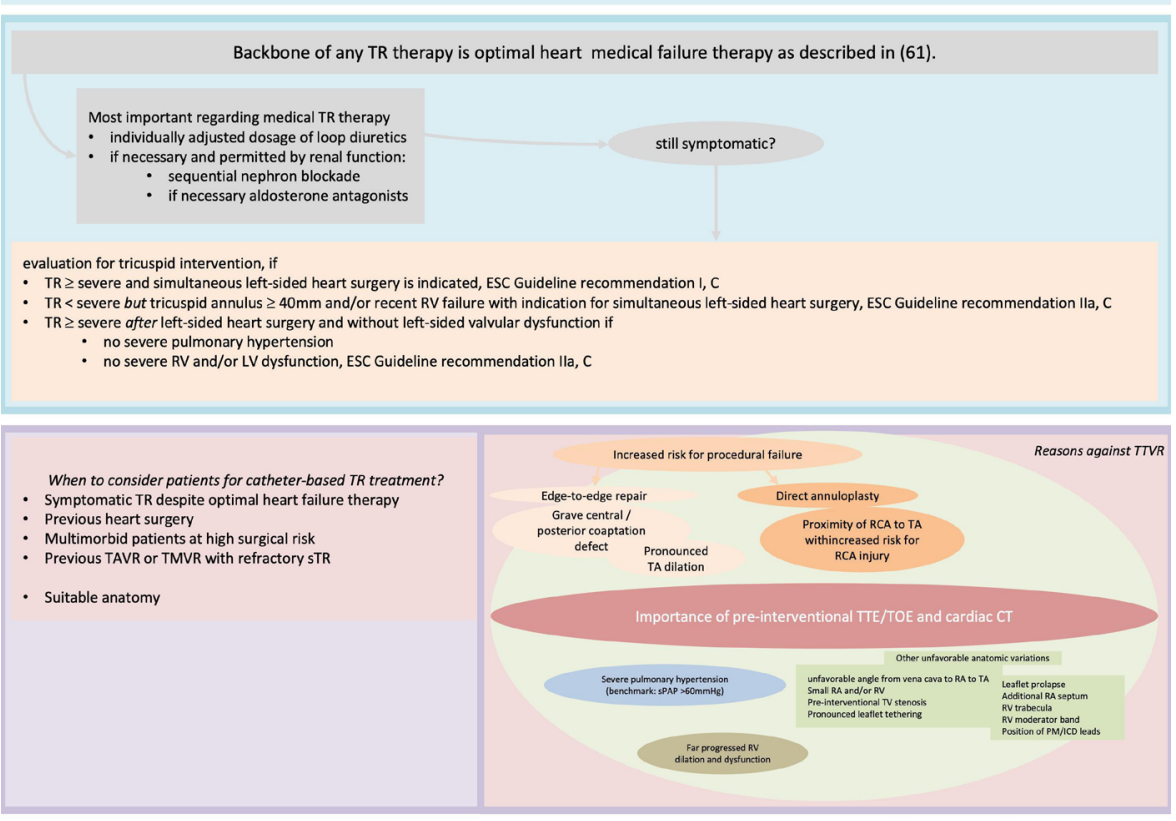

Figure 4 General considerations regarding TR therapy. The cornerstone of any TR therapy strategy is optimal heart failure therapy. Aside from diuretics (primarily loop diuretics and also sequential nephron blockade and aldosterone antagonists if permitted by renal function), no well-established guideline-recommendations for medical treatment exist specifically evaluated for TR therapy. Generally, TV repair is favoured over replacement. LV, left ventricle; RA, right atrium; RCA, right coronary artery; RV, right ventricle; sTR, secondary tricuspid regurgitation; TAVR, transcatheter aortic valve replacement; TMVR, transcatheter tricuspid valve repair/replacement; TOE, transoesophageal echocardiography; TTVR, transcatheter tricuspid valve repair/replacement; TV, tricuspid.

sudden cardiac death $(n=3)$ were the presence of a ventricular aneurysm and urinary 8-hydroxy-2'-deoxyguanosine (U-8-OHdG), a marker of oxidative
DNA damage that reflects the inflammatory activity of CS. ${ }^{3}$ In patients with an U-8-OHdG level $\geq 14.9 \mathrm{ng} / \mathrm{mg} \cdot \mathrm{Cr}$, the event rate per 100 patient-years was
0.6 (95\% CI 0.015 to 3.303) compared with 12.3 (95\% CI 6.739 to 20.681 ) in those with higher U-8OHdG levels (figure 3).

In an editorial, Franke and Mahajan ${ }^{4}$ comment that "the results of this study show some promise for the use of urinary 8 -OHdG in appropriate cohorts of patients with CS for risk stratification for primary prevention of SCD. Although this has exciting implications for the management of CS, particularly as urinary 8-OHdG is a simple, cost-effective test, larger, more robust studies are required to solidify its clinical use."

Another study in this issue of Heart looked at predictors of heart failure (HF) in patients with a diagnosis of sarcoidosis. ${ }^{5}$ In the Swedish National Patient Registry, the rate of $\mathrm{HF}$ was 2.2/1000 patient-years in 8574 sarcoidosis patients compared with $0.7 / 1000$ person-years in 84192 matched general population cases. The risk of $\mathrm{HF}$ in sarcoidosis patients was not associated with age, sex or treatment status and was not explained by a history of ischaemic heart disease. Independent predictors of HF in patients with sarcoidosis were diabetes, atrial fibrillation and ventricular arrhythmias, suggesting that the presence of arrhythmias indicated cardiac involvement by sarcoidosis.

The Education in Heart article ${ }^{6}$ in this issue summarises the pathophysiology of tricuspid regurgitation, the options for treatment, and the current criteria for patient selection and optimal timing of transcatheter tricuspid valve intervention (figure 4).

A review article on implications of cancer prior to and after heart transplantation $^{7}$ provides a comprehensive review of the literature and practical guidance for clinical practice. Up to a third of heart transplant recipients are diagnosed with cancer with 10 years of transplantation which represents a 1.5 -fold to 5 -fold higher risk than the general population. In patients with cancer prior to heart transplantation, the risk of recurrence declines with the length of time in remission being only $5 \%$ if $>5$ years free of cancer versus a $63 \%$ recurrence rates if $<1$ year in remission.

Another review article addresses the role of catheter ablation for management of atrial fibrillation in patients with heart failure. ${ }^{8}$ Atrial fibrillation and heart failure frequently coexist so that an integrated approach to treatment of both conditions is needed. 
Funding The authors have not declared a specific grant for this research from any funding agency in the public, commercial or not-for-profit sectors.

Competing interests None declared.

Patient and public involvement Patients and/or the public were not involved in the design, or conduct, or reporting, or dissemination plans of this research.

Patient consent for publication Not applicable.

Ethics approval This study does not involve human participants.

Provenance and peer review Commissioned; internally peer reviewed.

(c) Author(s) (or their employer(s)) 2022. No commercial re-use. See rights and permissions. Published by BMJ.

D Check for updates
To cite Otto CM. Heart 2022;108:407-409.

Heart 2022;108:407-409.

doi:10.1136/heartjnl-2022-320938

ORCID iD

Catherine M Otto http://orcid.org/0000-0002-05279392

\section{REFERENCES}

1 De Luca G, Algowhary M, Uguz B. COVID-19 pandemic, mechanical reperfusion and 30-day mortality in ST elevation myocardial infarction. Heart 2022;108:458-66.

2 Cammann VL, Templin C. The STEMI phenomenon during the COVID-19 pandemic: what is beneath the tip of the iceberg? Heart 2022;108:412-3.
3 Yoshitomi R, Kobayashi S, Yano Y. Enhanced oxidative stress and presence of ventricular aneurysm for risk prediction in cardiac sarcoidosis. Heart 2022;108:429-37.

4 Franke KB, Mahajan R. Cardiac oxidative stress: a potential tool for risk stratification in cardiac sarcoidosis. Heart 2022:108:410-1.

5 Rossides M, Kullberg S, Grunewald J. Risk and predictors of heart failure in sarcoidosis in a population-based cohort study from Sweden. Heart 2022;108:467-73.

6 Vogelhuber J, Weber M, Nickenig G. Transcatheter tricuspid intervention: ready for primetime? Heart 2022;108:479-91.

7 Mudigonda P, Berardi C, Chetram V. Implications of cancer prior to and after heart transplantation. Heart 2022;108:414-21.

8 Mulder BA, Rienstra M, Van Gelder IC. Update on management of atrial fibrillation in heart failure: a focus on ablation. Heart 2022;108:422-8. 